\title{
Face Recognition through Machine Learning of Periocular region
}

\author{
Ch Sai Chandana \\ Dept. of CSE \\ SNIST \\ Hyderabad, India
}

\author{
K Damodhar Rao \\ Associate Professor, Dept. of CSE \\ SNIST \\ Hyderabad, India
}

\author{
Dr. Prasanta Kumar Sahoo \\ Professor, Dept. of CSE \\ SNIST \\ Hyderabad, India
}

\begin{abstract}
Facial acknowledgment is a biometric software that is able to check an individual from an advanced picture or video outline by contrasting and investigating designs dependent on the individual's facial forms. Structuring the face acknowledgment frameworks that are invariant to aging is difficult as biometric frameworks endures severely because of subjects aging. None of the current strategies are keeping pace with human capacity in perceiving the comparability crosswise over two appearances. In this paper age invariant highlights are determined utilizing Local binary patterns and Gaussian naive Bayes is utilized a classifier for generating matching score. The experimentation is done on the FGNET database and the experimentation results show an acknowledgment precision of $96 \%$ with naive Bayes.
\end{abstract}

Keywords:-Local Binary Pattern, Periocular Region, ROI, Machine Learning, Navie Bayes

\section{INTRODUCTION}

Machine learning is a part of AI. The objective of AI for the most part is to comprehend the structure of information and fit that information into models that can be comprehended and used by individuals.

Face is most usually utilized biometric to perceive individuals. Face acknowledgment has gotten considerable consideration from scientists due to human exercises found in different utilizations of security like air terminal, criminal location, legal and so on. Contrasted with other biometric attributes like palm print, Iris, unique mark and so on. Face biometrics can be non-noisy. Face biometrics is a difficult field of examine with different restrictions forced for a machine face acknowledgment like varieties in head present, change in light, facial articulation, aging. Different approaches were proposed by scientists in defeating the confinements expressed.

Face acknowledgment can frequently demonstrate extraordinary compared to other biometrics since pictures can be taken without contacting or communicating with the individual being distinguished, and those pictures recorded and in a flash checked against existing databases.

Face acknowledgment can frequently demonstrate extraordinary compared to other biometrics since pictures can be taken without contacting or communicating with the individual being distinguished, and those pictures recorded and in a flash checked against existing databases.

Face acknowledgment offers superior, versatile answers for the most requesting ongoing or post-occasion necessities. With face surviellance, search, recognizable proof and verification all on a solitary stage, it tends to be handily coordinated into existing observation frameworks to extricate faces progressively, coordinate against a current database or watchlist and produce constant cautions to help diminish open dangers.

In a controlled domain, programmed facial acknowledgment frameworks give better exactness for the strategies that truly influenced by changeability of the particular persons aging designs. Appearance may change due to aging, lessening ease of use of facial datasets that are available. Biometric framework of aging procedure is yet another test for researchers due to applications of it, for example, scientific medication and the distinguishing proof of missing items that expect frameworks to be unchanging in the aging procedure. The primary confinement in the plan of these frameworks is the inaccessibility of a decent out of date database.

Periocular region is located near eye region i.e region around eye which may incorporate highlights like eyelashes, tear duct, skin texture, eyelids eyebrows, eye shape skin surface and some more. Periocular area verification framework is decent exchange among biometrics ofiris and face validation frameworks as it needsmore client participation. A surface classification strategy is proposed, it is LBP. It takes skin surface info from the picture by choosing binary pixel, setting limit neighboring every pixel.

Preprocessing strategies, for example, enhancement and denoising are done to the images to eliminate the noise present in images. The improvement method incorporates lighting elinimation, decreasing the effect of lighting changes.

\section{RELATED WORKS}

Anil K. Jain et al. has examined the feasibility of utilizing the periocular area as biometric trait. Global and local data are extracted from the periocular locale utilizing surface and point operators bringing about a list of feature set for matching this area. He has utilized three distinct matchers like LBP, GO, and SIFT for figuring out matching performance. He likewise considered the impacts of 
different factors, for example, division outward change is seen due to incorrect face detection. Results shows that the acknowledgment execution improves by considering the eyebrows in the perioculararea.

KishoreKumar Kamarajugaddaet.al recognized time of human utilizing AI calculations. Latest assessments done on huge data accumulated from maturing highlights shows presentation iamge based estimation of age is low and ought to be improvised. One of the commendable biometric characteristics for human affirmation or search is age of human. Right now periocular locale is utilized for highlight extraction. Right off the pre-processing is done on periocular district for age invariant highlights area is investigated by utilizing mixture approach that joins the both AI calculations SVM\& KNN to secure better outcomes.

An individual's face encounters numerous changes with the age that influence the working of biometric frameworks. The region near around eyes most probably will not change more across aging, it is used to design more stable age frameworks to feature enduring qualities.As periocular region is consistent it speeds up and decreases memory necessities, as it occupies only $25 \%$ of face. The proposed strategy utilizes the local binary pattern(LBP) in the periocular region to extract features from eye chisquare distance is used to calculate the matching score from the images in gallery. From the ROI imageLBP feature vectors are generated.

Face recognition using periocular region has gained more response in giving accurate results . It occupies only $25 \%$ part of the face so recognition time will be less and it will be easy to compare rather than comparing with whole face. It can be used in many real time appliactions like finding missing children, diagonise diseases etc.

\section{LIMITATIONS}

- Recognising the face using iris information cannot give proper results asimages captured from large distances may have less information of iris.

- Many machine learning algorithms are used in comparing or matchingthe faces/biometrics but some of them cannot give accurate results

- Some if the methods does not include eyebrows while considering periocularregion as it gives lowaccuracies.

\section{PROPOSED METHODOLOGY}

\section{Algorithm Used:}

\section{Naive Bayes:}

Generally speaking Bayes Theorem delineates the probability of an event, to be occur considering the events that has already occurred. Basically it perfectly suits for AI, as it is similar to what $\mathrm{AI}$ can do : makes desires that is for future reliant on the information that is related. Mathematically it can be represented as

$$
\mathrm{P}(\mathrm{a} / \mathrm{b})=\mathrm{P}(\mathrm{b} / \mathrm{a}) \mathrm{P}(\mathrm{a}) / \mathrm{P}(\mathrm{b})
$$

- Naive Bayes Algorithm is a quick, exceptionally adaptablecalculation

- Naive Bayes can be used for Binary and Multiclassorder.

- $\quad$ The Naïve Bayes Classifier can deliver very exact characterization results with a base preparing time when contrasted with traditional directed or unaided learning calculations.

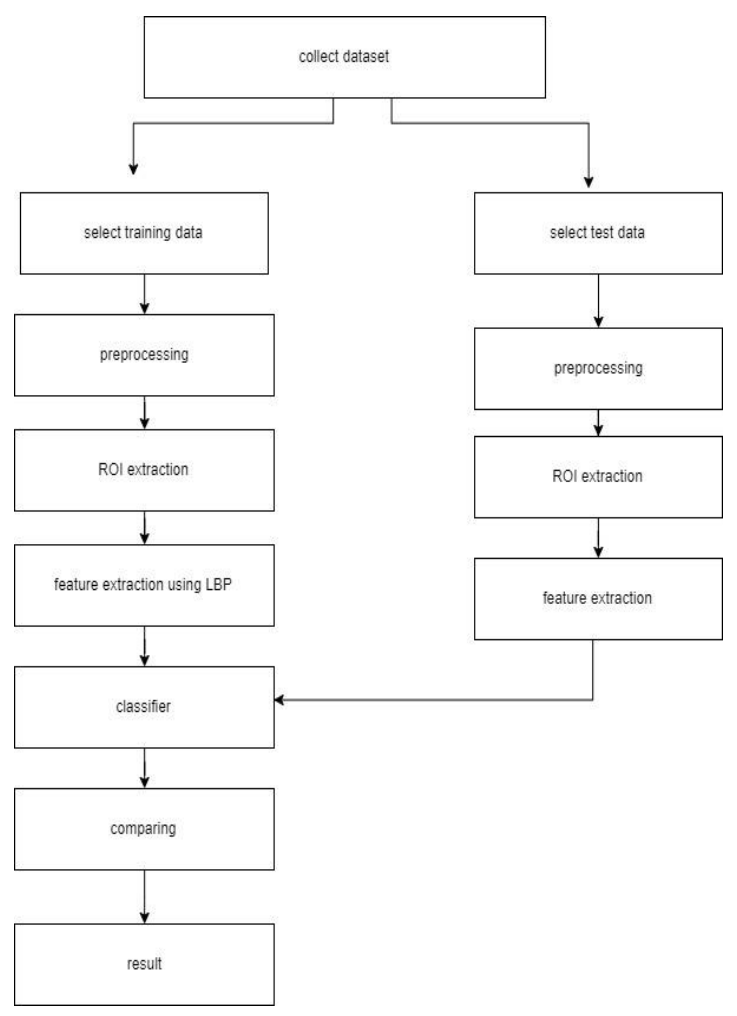

Fig : Data Flow Diagram

FGNET dataset is the dataset of all the human face pictures in the pictures organizer. We have an aggregate of 1000 pictures in the dataset. All the pictures are in the JPG format.Each picture is named with various name for instance 006A28.jpg implies this is the 'sixth' individual's picture when he/she was 28 years of age. 'A' is short for Age.

This algorithm is used to verify how accurately images of face are matching. The FGNET dataset that we have taken in this paper comprises of 1000 samples and we divide them into train and test. Here Navie Bayes algorithm analyzes the training and testing images that we have selected and it provides the accuracy. In this paper the dataset we have taken consists of 1000 samples of face images and these are in the jpg format. 
After splitting the dataset into training and testing ,the training process images are collected from the FGNET dataset. We have to set an ID for every image with the name or any number. Every individual should have different id. So during training algorithm uses this information and it provides us the result. Preprocessing is done to the images to eliminate noise present in images. After preprocessing ROI extraction is done to detect eye region for LBP computation. The images that are trained are used for LBP computation.It extracts the features from eye. LBP computation creates histograms from the eye region that is detected using $\mathrm{ROI}$.

In the next step face recognition is done for this purpose we have to consider the test images and the same process repeats as in the training and the classifier we have used compares the result and gives the accuracy. The classifier gives the result by comparing the histograms created for training and testing images.

\section{RESULTS}

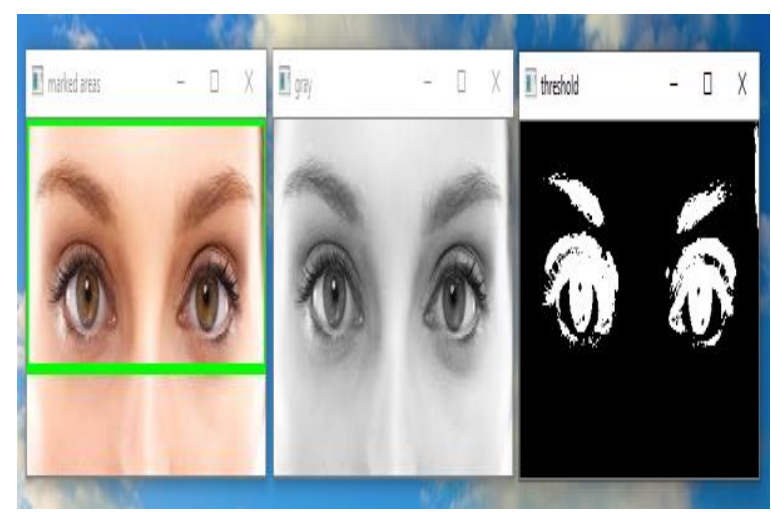

Fig : ROI Detection

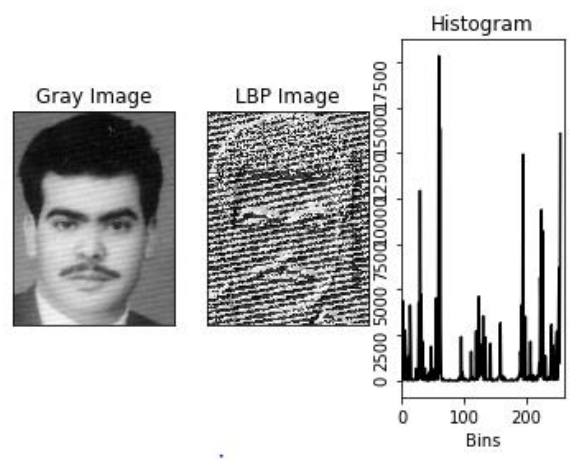

Fig: LBP Histogram

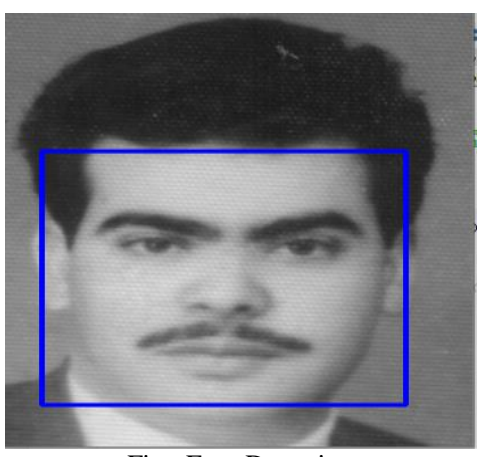

Fig : Face Detection

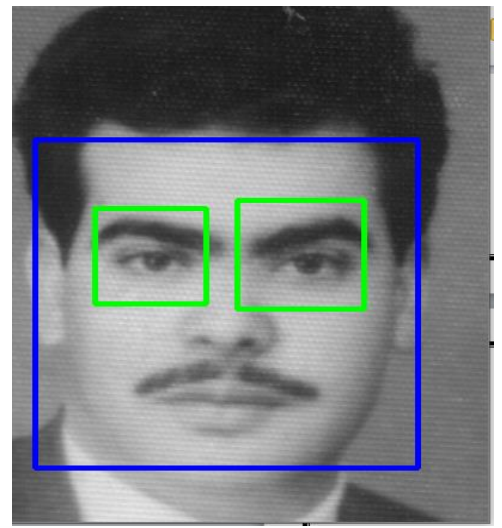

Fig : ROI Detection

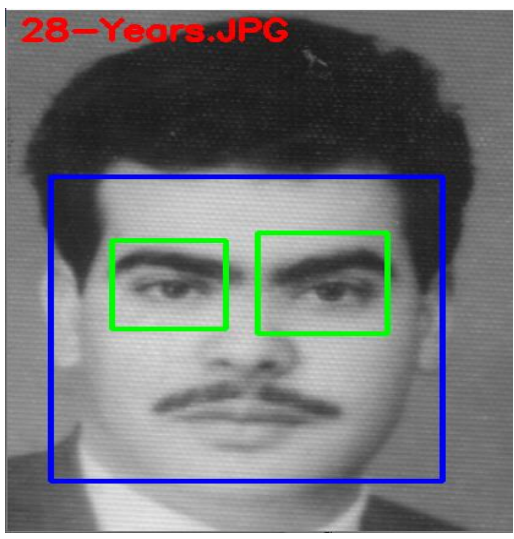

Fig : Detecting Age by periocular region

\begin{tabular}{|l|l|l|}
\hline Dataset & Algorithm & Accuracy \\
\hline $\begin{array}{l}\text { FGNET } \\
\text { dataset }\end{array}$ & Naive Bayes & $96 \%$ \\
\hline $\begin{array}{l}\text { FGNET } \\
\text { dataset }\end{array}$ & Logistic regression & $40 \%$ \\
\hline
\end{tabular}

Table 1 : comparing two algorithms.

\section{CONCLUSION}

There were many approaches for face recognition by considering iris ,face, etc. but it will be difficult for identifying correctly because when the camera is far away from the person it could not capture image correctly with the iris and also it causes the low resolution images which fails in predicting the image correctly. There comes another approach known as periocular region as this region contains most discriminating capability when compared to other parts by considering features like eyebrows, eye lashes, eye lids, skin texture etc. In this research work we used Gaussian naive Bayes classifier which gives $95 \%$ accuracy when compared to other past research works in this area. 


\section{REFERENCES}

[1] Kumar KK, Trinatha Rao P (2018) Biometric identification using the periocular region. In: 2nd international conference on information and communication technology for intelligent systems, Springer SIST Series, vol 84. Springer, Cham, pp 619-628

[2] KumarKK,Pavani M(2017)LBPbased biometric identification using the periocular region.In: IEEE 8th annual information technology, electronics and mobile communication conference (IEMCON). Vancouver, BC, pp 204-209

[3] Joshi A, Gangwar A, Sharma R, Saquib Z (2012) Periocular feature extraction based on LBP and DLDA. In: Advances in computer science, engineering \&applications, volume 166 of advances in intelligent and soft computing. Springer, pp 10231033

[4] Kumar KK, Trinatha Rao P (2018) Periocular region based biometric identification using the local descriptors. In: Intelligent computing and information and communication. advances in intelligent systems and computing, vol 673 . Springer, Singapore, pp 341-351

[5] Woodard DL, Pundlik SJ, Lyle JR, Miller PR (2010) Periocular region appearance cues for biometric identification. In: 2010 IEEE computer society conference on computer vision and pattern recognition workshops (CVPRW). IEEE, pp $162-169$

[6] Lyle JR, Miller PE, Pundlik SJ, Woodard DL (2010) Soft biometric classification using periocular region features. In: 2010 fourth IEEE international conference on biometrics: theory applications and systems (BTAS). IEEE, pp 1-7

[7] Mahalingam G, Kambhamettu C (2010) Age invariant face recognition using graph matching. In: 2010 fourth IEEE international conference on biometrics: theory applications and systems (BTAS). IEEE, pp 1-7

[8] Park U, Jillela R, Ross A, Jain A (2011) Periocular biometrics in the visible spectrum. IEEE Trans Inf Forensics Secure 6(1):96-106

[9] Park Unsang, Tong Yiying, Jain Anil K (2010) Age-invariant face recognition. IEEE Trans Pattern Anal Mach Intell 32(5):947-954

[10] Joshi A, Gangwar A, Sharma R, Saquib Z (2012) Periocular feature extraction based on LBP and DLDA. In: Advances in computer science, engineering \& applications, volume 166 of advances in intelligent and soft computing. Springer, pp 102310 